\title{
SOME PRACTICAL PROCEDURES FOR THE SOLUTION OF NONLINEAR FINITE ELEMENT EQUATIONS
}

\author{
Klaus Jürgen BATHE and Arthur P. CIMENTO \\ Department of Mechanical Engineering, Massachusetts Institute of Technology, \\ Cambridge, Massachusetts 02139, USA
}

Received 21 March 1979

\begin{abstract}
Procedures for the solution of incremental finite element equations in practical nonlinear analysis are described and evaluated. The methods discussed are employed in static analysis and in dynamic analysis using implicit time integration. The solution procedures are implemented, and practical guidelines for their use are given.
\end{abstract}

\section{Introduction}

During recent years the nonlinear finite element analysis of static and dynamic problems has been an area of growing interest in engineering. Various finite element computer programs are currently in use for the analyses of complex nonlinear problems [1]. Basically, these analyses involve three steps: the selection of a representative finite element model, the analysis of the model and the interpretation of the results. Surely, in engineering practice the selection of an appropriate finite element model and the corresponding interpretation of the results are crucial, but a reliable and accurate response prediction of the model is essential in order that the analysis results can be used with confidence.

Unfortunately, considering the present nonlinear analysis procedures, the accurate analysis of a finite element model can present very great difficulties. The cost of analysis can be high, but a more serious factor is that considerable knowledge and judgment by the user may be required to assure a stable and accurate solution. Hence, there is a great need for solution algorithms with increased accuracy and stability properties.

In general, a nonlinear static and dynamic finite element analysis is most effectively performed using an incremental formulation, in which the static and kinematic variables are updated incrementally corresponding to successive load steps (or time steps in dynamics) in order to trace out the complete solution path [1]-[8]. In this solution it is important that the governing finite element equations are satisfied in each load step to sufficient accuracy because otherwise solution errors can accumulate that can lead to gross and undetectable errors and even to solution instabilities [2]-[11].

An accurate solution of the nonlinear finite element equations can always be expected if the load increments per step are made sufficiently small, but such a solution can result in many incremental steps that render the analysis of a large finite element system prohibitively 
expensive. In order to solve the nonlinear finite element equations efficiently while maintaining control on the accuracy of the solution, larger load steps may need to be employed with iteration that assures the accurate solution of the nonlinear equations. However, the use of iteration can introduce some other difficulties. The convergence process may be slow, requiring a large number of iterations that can again result in a high solution cost. Also, some iterative methods do not converge for certain types of problems or for large load increments. In practice, such difficulties can result in costly numerical experimentations, which inhibit the wide use of nonlinear finite element analysis.

The objective of this paper is to report on what we believe at present to be some effective, practical procedures for the incremental solution of nonlinear finite element equations. We assume that a solution exists with finite solution variables to the nonlinear problem which is analyzed, and if there are multiple solutions (e.g. because of limit and bifurcation points), all that the calculation can yield is "a" solution in the multidimensional solution space. Considering dynamic analysis, we also assume that an implicit time integration scheme and a time step $\Delta t$ have already been selected, and we only consider the accurate solution of the timewise discretized equilibrium equations. Since these equations are linear in explicit time integration (e.g. using the central difference method [8], [16]), we are only concerned with implicit integration of dynamic response.

We first briefly summarize the general analysis approach employed and the incremental finite element equations that need to be solved. We consider static analysis and dynamic analysis using the trapezoidal rule for the time integration. We then describe the procedures that are the most promising candidates for the iterative solution of the incremental equilibrium equations: (a) modified Newton iteration with Aitken acceleration and a divergence procedure and (b) the Broyden-Fletcher-Goldfarb-Shanno (BFGS) method [12]-[15]. Next we discuss the importance of using appropriate convergence criteria to stop the iteration, and we propose specific ways to measure convergence. The iterative schemes have been implemented in the ADINA computer program, and finally, we give the results of some demonstrative sample solutions. These results illustrate the relative effectiveness of the solution techniques employed and, together with additional experiences, lead to the presentation of some practical guidelines for the selection of the appropriate solution scheme corresponding to a specific nonlinear analysis.

\section{Incremental finite element equations}

As already mentioned in the previous section, a general geometric and material nonlinear finite element analysis is performed most effectively by use of an incremental formulation of the equations of motion.

In the notation of [4]-[8] the incremental finite element equations that govern the response of the finite element system in static analysis are

$$
{ }^{t} \boldsymbol{K} \boldsymbol{U}={ }^{t+\Delta t} \boldsymbol{R}-{ }^{t} \boldsymbol{F}
$$

where ${ }^{t} \boldsymbol{K}=$ tangent stiffness matrix corresponding to the configuration of the system at time $t$; $\boldsymbol{U}=$ vector of nodal point incremental displacements, i.e. $\boldsymbol{U}={ }^{t+\Delta t} \boldsymbol{U}-{ }^{t} \boldsymbol{U} ;{ }^{t+\Delta t} \boldsymbol{R}=$ vector of 
externally applied nodal point loads corresponding to time $t+\Delta t$; and $\boldsymbol{F}=$ vector of nodal point forces corresponding to the internal element stresses at time $t$.

As indicated in eq. (1) and illustrated in fig. 1, we use the discrete time increment $\Delta t$ to denote a load increment. If the material properties are time-dependent (such as in creep analysis), the time increments must be chosen judiciously for stability and accuracy; otherwise, the discrete time points considered simply denote the load levels.

With reference to fig. 1 it should also be noted that the time step magnitudes need not be identical throughout the incremental analysis; indeed, a variation of the time step magnitudes can be particularly effective in the calculation of creep response.

Considering now dynamic analysis using implicit time integration, the governing incremental finite element equations of motion are

$$
{ }^{t} \boldsymbol{K} \boldsymbol{U}={ }^{t+\Delta t} \boldsymbol{R}-{ }^{t} \boldsymbol{F}-\boldsymbol{M}^{t+\Delta t} \ddot{\boldsymbol{U}},
$$

where the variables of eq. (1) are used and $\boldsymbol{M}=$ mass matrix, and ${ }^{t+\Delta t} \ddot{\boldsymbol{U}}=$ vector of nodal point accelerations corresponding to time $t+\Delta t$.

In eq. (2) we did not inçlude damping effects expressed by a damping matrix times the nodal point velocities, but this effect could easily be included in an analogous manner to the inertia effects, provided the damping matrix can be constructed [16].

Various implicit time integration methods are presently in use. We consider here the trapezoidal rule because of its relatively good stability and accuracy characteristics [9]-[11], [17], but other integration schemes would be employed in an analogous manner. The basic assumptions of the trapezoidal rule are

$$
{ }^{t+\Delta t} \dot{U}={ }^{t} \dot{U}+\frac{\Delta t}{2}\left({ }^{t+\Delta t} \ddot{U}+{ }^{t} \ddot{U}\right)
$$

and

$$
{ }^{t+\Delta t} \boldsymbol{U}={ }^{\prime} \boldsymbol{U}+\frac{\Delta t}{2}\left({ }^{t+\Delta t} \dot{\boldsymbol{U}}+{ }^{\prime} \dot{\boldsymbol{U}}\right)
$$

Using eqs. (3) and the relation $U={ }^{t+\Delta t} U-{ }^{t} U$, eq. (2) gives

$$
\left({ }^{\prime} \boldsymbol{K}+\frac{4}{(\Delta t)^{2}} \boldsymbol{M}\right) \boldsymbol{U}={ }^{t+\Delta t} \boldsymbol{R}-{ }^{\prime} \boldsymbol{F}+\boldsymbol{M}\left(\frac{4}{\Delta t} t \dot{U}+' \ddot{U}\right) .
$$

Eq. (4) is solved recursively for all time steps, where, as in static analysis, the time step increments can vary during the incremental solution.

Eqs. (1) and (2) are derived by linearizing the system response about the configuration at time $t$. It was repeatedly pointed out that the errors in this linearization can be expected to be small provided the load increments or time steps are small enough. However, in general, because of cost considerations an analyst must endeavor to employ as large load and time steps as possible, and in such a case the errors resulting from the linearization may not be negligible, and their accumulation can lead to gross errors or instability of the solution. The 

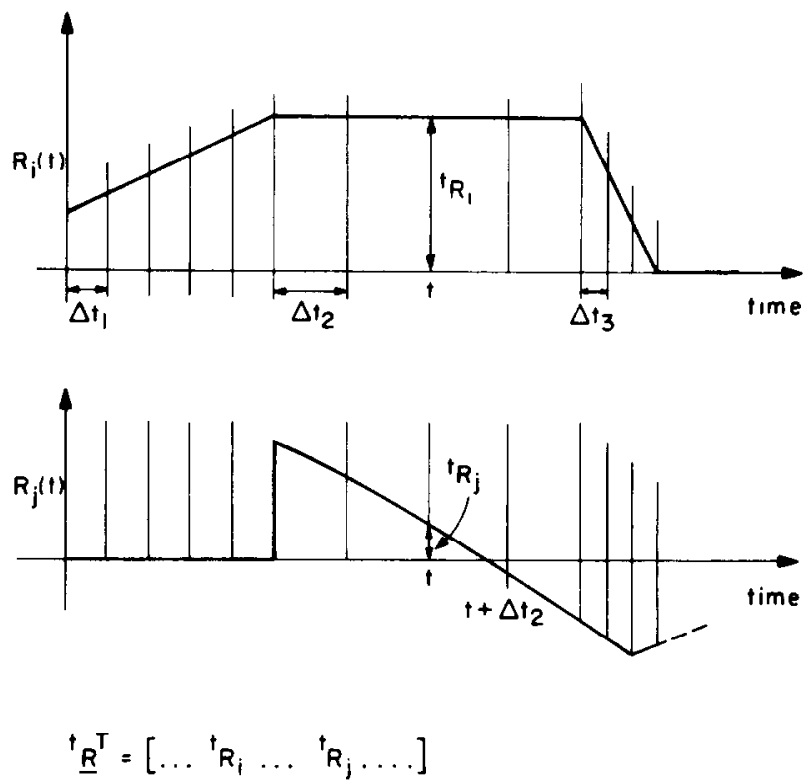

Fig. 1. Evaluation of externally applied nodal point load vector ${ }^{2} \boldsymbol{R}$ at time $t$.

accumulation of the solution errors can be particularly serious in dynamic analysis, where relatively small increments in errors at the individual discrete time points can rapidly cause a solution instability [5], [18]. As concluded earlier, when larger load steps or time steps are used, iteration for system equilibrium at the discrete time points is necessary although the accurate solution of the timewise discretized equilibrium equations does not strictly always imply a stable solution [10], [17]. Since the analyst does not generally know whether the load or time step chosen is "small" or "large", based on accuracy considerations alone, iteration in the solution of the system equilibrium equations is desirable at all load or time steps. This iteration should be as cost-effective as possible; i.e. rapid convergence should occur with the total number of numerical operations required in the iteration as small as possible.

\section{Iteration procedures for solution of equilibrium equations}

The basic equations to be solved in nonlinear analysis are (corresponding to time $t+\Delta t$ )

$$
{ }^{t+\Delta t} \tilde{\boldsymbol{R}}\left(\boldsymbol{U}^{*}\right)-{ }^{t+\Delta t} \boldsymbol{F}\left(\boldsymbol{U}^{*}\right)=\boldsymbol{o},
$$

where

$$
{ }^{t+\Delta t} \tilde{\boldsymbol{R}}\left(\boldsymbol{U}^{*}\right)={ }^{t+\Delta t} \boldsymbol{R}-\boldsymbol{M}^{t+\Delta t} \ddot{\boldsymbol{U}}^{*}
$$

Eq. (5) holds for the dynamic analysis considered in eq. (2), and for the static analysis considered in eq. (1) if the mass effects are neglected. Also, if the load or time increments are small, eqs. (1) and (2) yield an approximate solution to eq. (5). However, we now consider 
large load and time increments where we need to solve eq. (5) by iteration-note then that the first iteration in the equations below reduces to the solutions of eqs. (1) and (2).

\subsection{The Newton-Raphson iteration}

The most frequently used iteration schemes for the solution of nonlinear finite element equations are some form of Newton-Raphson iteration [2]-[7], [19]. The equilibrium requirements amount to finding the solution of the equations

$$
\boldsymbol{f}\left(\boldsymbol{U}^{*}\right)=\boldsymbol{o},
$$

where

$$
f\left(U^{*}\right)={ }^{t+\Delta t} \tilde{R}\left(U^{*}\right){ }^{-{ }^{t+\Delta t}} \boldsymbol{F}\left(U^{*}\right)
$$

A Taylor series expansion of $\boldsymbol{f}\left(\boldsymbol{U}^{*}\right)$ about the solution $\boldsymbol{U}^{*}$ gives

$$
\boldsymbol{f}\left(\boldsymbol{U}^{*}\right)=\boldsymbol{f}\left({ }^{t+\Delta t} \boldsymbol{U}^{(i-1)}\right)+\left[\left.\frac{\partial \boldsymbol{f}}{\partial \boldsymbol{U}}\right|_{{ }^{++\Delta t} \boldsymbol{U}^{(i-1)}}\right]\left(\boldsymbol{U}^{*}-{ }^{t+\Delta t} \boldsymbol{U}^{(i-1)}\right),
$$

where higher-order terms are neglected, and the superscript $(i-1)$ is used to denote the $(i-1)$ st approximation to the solution vector $U^{*}$. Substituting from eq. (8) into eq. (9) and using the condition in eq. (7), we obtain

$$
{ }^{t+\Delta t} \tilde{\boldsymbol{R}}^{(i-1)}-{ }^{t+\Delta t} \boldsymbol{F}^{(i-1)}+\left[\left.\frac{\partial \tilde{\boldsymbol{R}}}{\partial \boldsymbol{U}}\right|_{{ }^{1+\Delta t} \boldsymbol{U}^{(i-1)}}-\left.\frac{\partial \boldsymbol{F}}{\partial \boldsymbol{U}}\right|_{{ }^{t+\Delta t} U^{(i-1)}}\right]\left(\boldsymbol{U}^{*}-{ }^{t+\Delta t} \boldsymbol{U}^{(i-1)}\right)=\boldsymbol{o}
$$

where

$$
{ }^{t+\Delta t} \tilde{\boldsymbol{R}}^{(i-1)}={ }^{t+\Delta t} \boldsymbol{R}-\boldsymbol{M}^{t+\Delta t} \ddot{U}^{(i-1)}
$$

We now define

$$
\Delta U^{(i)}=U^{*}-{ }^{t+\Delta t} U^{(i-1)}
$$

and recognize that

$$
\left.\frac{\partial \boldsymbol{F}}{\partial \boldsymbol{U}}\right|_{{ }_{t+\Delta t} \boldsymbol{U}^{(i-1)}}={ }^{t+\Delta t} \boldsymbol{K}^{(i-1)},
$$

where ${ }^{t+\Delta t} K^{(i-1)}$ is the tangent stiffness matrix in iteration $i-1$. Also, using the trapezoidal rule for time integration, we have

$$
\left.\frac{\partial \tilde{\boldsymbol{R}}}{\partial \tilde{U}}\right|_{{ }^{t+\Delta U^{\prime}} \boldsymbol{U}^{(i-1)}}=-\frac{4}{(\Delta t)^{2}} \boldsymbol{M}
$$


Thus eq. (10) yields

$$
{ }^{t+\Delta t} \hat{\boldsymbol{K}}^{(i-1)} \Delta \boldsymbol{U}^{(i)}={ }^{t+\Delta t} \boldsymbol{R}-{ }^{t+\Delta t} \boldsymbol{F}^{(i-1)}-\boldsymbol{M}\left\{\frac{4}{(\Delta t)^{2}}\left({ }^{t+\Delta t} \boldsymbol{U}^{(i-1)}-{ }^{t} \boldsymbol{U}\right)-\frac{4}{\Delta t} t \dot{\boldsymbol{U}}-{ }^{t} \ddot{\boldsymbol{U}}\right\},
$$

where

$$
{ }^{t+\Delta t} \hat{\boldsymbol{K}}^{(i-1)}={ }^{t+\Delta t} \boldsymbol{K}^{(i-1)}+\frac{4}{(\Delta t)^{2}} \boldsymbol{M} .
$$

Since eq. (10) represents only a Taylor series approximation to $U^{*}$, the displacement increment correction is used to obtain the next displacement approximation

$$
{ }^{t+\Delta t} U^{(i)}={ }^{t+\Delta t} U^{(i-1)}+\Delta U^{(i)} .
$$

Eqs. (15) and (17) constitute the Newton-Raphson solution of eq. (5), with the initial conditions

$$
{ }^{t+\Delta t} \hat{\boldsymbol{K}}^{(0)}={ }^{t} \hat{\boldsymbol{K}}, \quad{ }^{t+\Delta t} \boldsymbol{F}^{(0)}={ }^{t} \boldsymbol{F} \quad \text { and } \quad{ }^{t+\Delta t} \boldsymbol{U}^{(0)}={ }^{t} \boldsymbol{U}
$$

The iteration using eqs. (15) and (17) continues until appropriate termination criteria are satisfied (see section 4).

Although the use of the full Newton iteration of eqs. (15)-(17) can be efficient in some specific nonlinear analyses, the use of the method is generally not very effective in general geometric and material nonlinear response calculations. Considering the updating and factorizing of the effective stiffness matrix ${ }^{t+\Delta t} \hat{K}^{(i-1)}$ in each iteration, this process is computationally expensive and, for solution effectiveness, requires the use of relatively large load increments. However, in material nonlinear analysis or dynamic nonlinear response calculations the load (or time) steps that can be employed are restricted in size by other stability and accuracy considerations [5]-[11]. For example, in elastic-plastic analysis, proportional loading (unloading) is generally assumed during a load step, which therefore has to be reasonably small. Also, inaccurate approximations to the displacements during the iteration can introduce significant errors because the material properties depend on the history of the stresses and strains. For these reasons some modification of the full Newton algorithm is generally effective.

One such modification is to use the initial stiffness matrix ${ }^{0} \hat{\boldsymbol{K}}$ in eq. (15) and thus operate on the equations

$$
{ }^{0} \hat{\boldsymbol{K}} \Delta \boldsymbol{U}^{(i)}={ }^{t+\Delta t} \tilde{\boldsymbol{R}}^{(i-1)}-{ }^{t+\Delta t} \boldsymbol{F}^{(i-1)} .
$$

In eq. (18) only the matrix ${ }^{0} \hat{\boldsymbol{K}}$ need be factorized; thus the expense of recalculating and factorizing the coefficient matrix in eq. (15) many times is avoided. This "initial stress" method corresponds to a linearization of the response about the initial configuration of the finite element system. For problems with significant nonlinearities, and in particular when the system stiffens during the response, this linearization can lead to a very slow convergence in 
the iteration, or the iteration may even diverge. In order to accelerate the convergence and prevent divergence of the solution for slowly stiffening problems (see discussion below), it may be effective to use the modified Newton-Raphson iteration. In this method we calculate from time to time a new tangent stiffness matrix and operate on the equations

$$
{ }^{\tau} \hat{\boldsymbol{K}} \Delta \boldsymbol{U}^{(i)}={ }^{t+\Delta t} \tilde{\boldsymbol{R}}^{(i-1)}-{ }^{t+\Delta t} \boldsymbol{F}^{(i-1)},
$$

where $\tau$ corresponds to one of the accepted equilibrium configurations at times $0, \Delta t, 2 \Delta t, \ldots$ or $t$. In eq. (19) the vector ${ }^{t+\Delta t} F^{(i-1)}$ is evaluated from the stresses that correspond to time $t+\Delta t$ and iteration $i-1$. Considering a point within a finite element, this stress evaluation can be written as

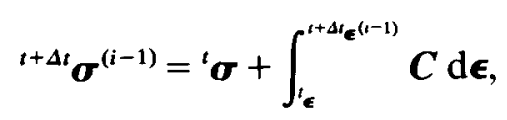

where ${ }^{t} \boldsymbol{\sigma}$ are the stresses corresponding to the last accepted equilibrium configuration, ' $\boldsymbol{\epsilon}$ denotes the strains at time $t$, and $\boldsymbol{C}$ is the stress-strain matrix of the material, which in general is not constant during the integration. It is important in eq. (20) that the integration always be performed from the last accepted or converged strains to the presently calculated strains. Thus the final converged results are not affected by the errors in the displacements and strains that were encountered during the iteration.

The modified Newton method involves fewer stiffness reformations than full Newton iteration, and the stiffness matrix update is based on an accepted equilibrium configuration. The choice of time steps when the stiffness matrix should be updated depends on the degree of nonlinearity in the system response, i.e. the more nonlinear the response, the more often the updating should be done. Without any a priori knowledge of the system behavior it may be most efficient to update the stiffness at the start of every time step, in which case $\tau=t$ in eq. (19).

\subsection{Slow convergence and divergence of modified Newton iteration}

In its application to nonlinear structural analysis the modified Newton method presents practical difficulties. The two problems of slow convergence and divergence are most frequently encountered.

A large number of iterations will be required for convergence when there is a sudden softening in the system during a time increment. Fig. 2 illustrates this phenomenon for a one-dimensional case. Such a force-displacement relationship is typical of an elastic-plastic material, where ${ }^{i+\Delta t} K /{ }^{t} K=E_{T} / E$. As this ratio tends to zero and/or the load increment ${ }^{t+\Delta t} R-{ }^{t} F$ becomes large, the number of iterations required for convergence becomes very large. In practice, where some limit is placed on the number of iterations that can be used, the solution would terminate too early. In order to make the modified Newton method effective in cases of slow convergence, some acceleration scheme should be employed.

A commonly used acceleration scheme is the one employed by Aitken for eigenvalue problems [20]. With Aitken acceleration eq. (17) becomes

$$
{ }^{t+\Delta t} \boldsymbol{U}^{(i)}={ }^{t+\Delta t} U^{(i-1)}+\boldsymbol{\alpha}^{(i-1)} \Delta \boldsymbol{U}^{(i)}
$$



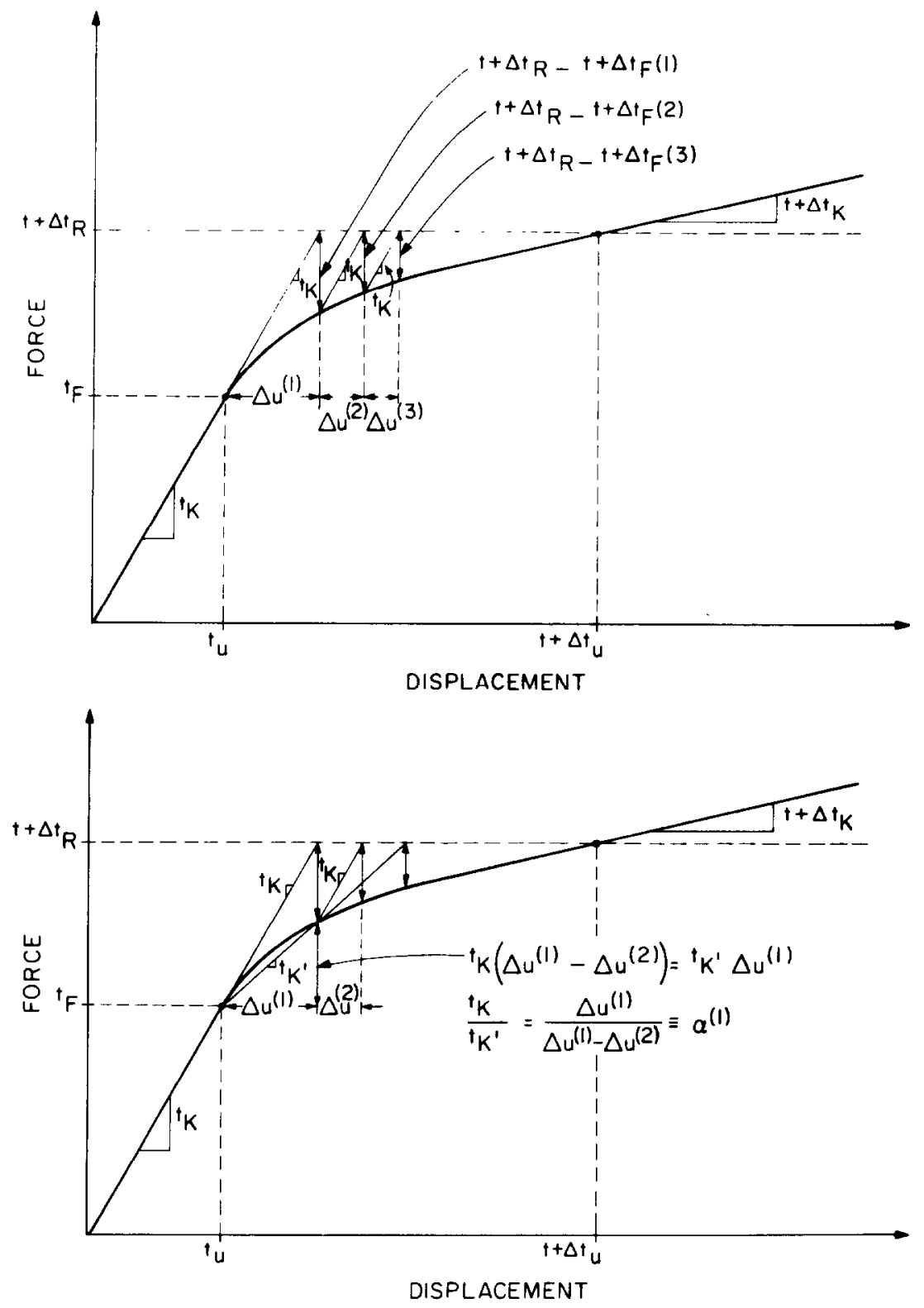

Fig. 2. Slow convergence and acceleration of the modified Newton iteration in a one-dimensional example.

where $\boldsymbol{\alpha}^{(i-1)}$ is an $n \times n$ diagonal coefficient matrix containing the acceleration factors, and $n$ is the number of degrees of freedom in the system. The acceleration factor for each degree of freedom is given by

$$
\alpha_{i}^{(i-1)}=\frac{\Delta U_{i}^{(i-1)}}{\left(\Delta U_{i}^{(i-1)}-\Delta U_{i}^{(i)}\right)^{.}}
$$

As shown in fig. 2, the accelerator gives an estimate of the ratio ' $K /{ }^{\prime} K{ }^{\prime}$ based on the information obtained in the iteration. 
Difficulties with Aitken acceleration can arise when the denominator in eq. (22) is small for some degrees of freedom. Also, since the accelerator estimates the ratio of the original tangent stiffness to the local secant stiffness using the difference between successive out-of-balance load terms (see fig. 2), Aitken acceleration can only be applied every other iteration to avoid inaccurate estimates of this ratio. To circumvent these problems, modifications of Aitken's accelerator have been developed [21], [22], but for a general analysis it has not been demonstrated that these versions accelerate convergence any better than the original Aitken method.

Another difficulty with modified Newton iteration arises when the out-of-balance loads ${ }^{t+\Delta t} \tilde{\boldsymbol{R}}^{(i)}-{ }^{t+\Delta t} \boldsymbol{F}^{(i)}$ increase during the solution, thus signalling divergence from the solution. Iteration divergence can occur whenever the system stiffens during the solution increment.

Two types of stiffening should be distinguished. Slow stiffening, illustrated by curve I in fig. 3 , can typically occur when geometric nonlinearities are included in the analysis. In these cases, choosing a smaller load increment so that the linearization about time $t$ yields a close enough approximation to the stiffness matrix at time $t+\Delta t$ is sufficient to obtain convergence. Sudden stiffening, illustrated by curve II in fig. 3, can occur when unloading of a nonlinear material (e.g. elastic-plastic material) is predicted in the solution. In this case the solution converges in dynamic analysis provided the time step $\Delta t$ is small enough [12], but in static analysis a smaller load step is not sufficient to obtain convergence [7]. In static analysis the stiffness matrix needs to be reformed based on the elastic material properties at time $t$, and this stiffness matrix must be employed in the solution of eq. (19).

Using the above concept, the following divergence scheme has been incorporated into the modified Newton-Raphson iteration in static analysis. When divergence is detected (see section 4 for the termination criterion), the iteration is halted, and an elastic stiffness matrix, ${ }^{\prime} \boldsymbol{K}^{E}$ based on the geometry at time $t$, is calculated to handle problems involving sudden stiffening. The load increment is also scaled by a factor $\lambda_{1}\left(\lambda_{1} \leq 1\right)$ to handle divergence due to additional slow stiffening; thus eq. (19) is modified to

$$
{ }^{t} \boldsymbol{K}^{\mathrm{E}} \Delta \boldsymbol{U}^{(i)}={ }^{t+\Delta t} \boldsymbol{R}-{ }^{t+\lambda_{1} \Delta t} \boldsymbol{F}^{(i-1)}-\left(1-\lambda_{1}\right)\left({ }^{t+\Delta t} \boldsymbol{R}-\boldsymbol{F}\right),
$$

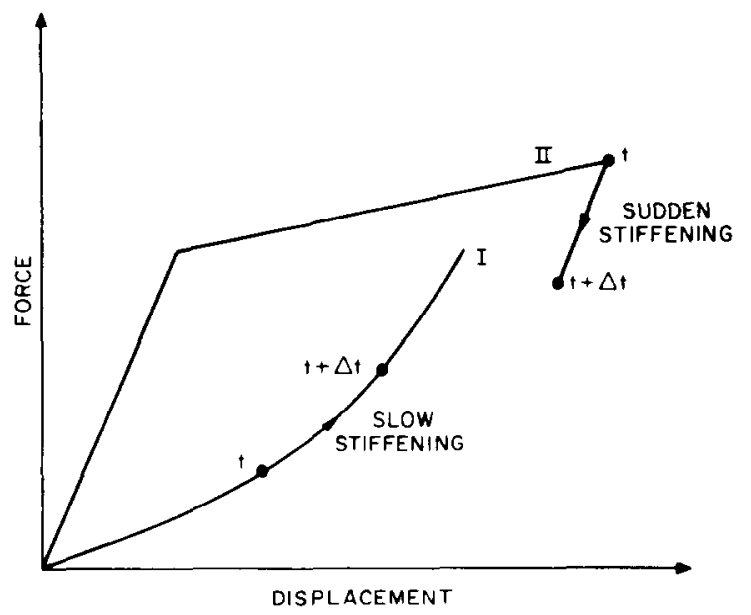

Fig. 3. Force-displacement relations leading to divergence in the modified Newton iteration. 
and we iterate until the solution at the intermediate time $t+\lambda_{1} \Delta t$ is determined. If divergence is still detected, $\lambda_{1}$ is continually chosen to be some smaller value until convergence is obtained (with some lower limit on $\lambda_{1}$ specified). After a solution has been obtained, a second factor $\lambda_{2}$ is chosen, the stiffness matrix is re-formed about the configuration at time $t+\lambda_{1} \Delta t$, and the displacements at time $t+\left(\lambda_{1}+\lambda_{2}\right) \Delta t$ are found in an analogous manner. This solution continues until

$$
\sum_{k=1}^{m} \lambda_{k}=1
$$

with some upper limit imposed on $m$.

The selection of $\lambda_{k}$ in this procedure is based on the number of iterations required for convergence with $\lambda_{k-1}$. The initial estimate of $\lambda_{1}$ is taken as 0.5 to minimize the number of intermediate solutions if elastic unloading is present and re-forming the stiffness is all that is necessary to obtain convergence. Thereafter, $\lambda_{k}$ is set to 0.5 for rapid convergence (less than 4 iterations were used for convergence), 0.25 for moderately fast convergence ( 4 to 12 iterations), and 0.0625 for slow convergence (more than 12 iterations). In the case of rapid convergence the stiffness matrix is not re-formed for the next intermediate solution. Although the step size selection procedure is empirical, the method is rational, and its effectiveness has been demonstrated in a large number of analyses. A more theoretical approach toward an adaptive step-size selection based on the convergence rate of a full Newton iteration scheme has been proposed by Schmidt [23], but when applied to the modified Newton method it was found to be less effective than the simple procedure outlined above. As another mechanism the current stiffness parameter discussed in [24] may also be useful in establishing an automatic load incrementation scheme. Our divergence procedure does not guarantee convergence because an incremental load step in the scheme may still be too large, but the procedure does attempt to correct the more common reasons for divergence of the modified Newton iteration.

\subsection{Matrix updating iterative methods}

As an alternative to forms of Newton iteration, a class of methods known as matrix update methods or quasi-Newton methods have been developed for iteration on nonlinear systems of equations. These methods involve updating the coefficient matrix (or rather its inverse) to provide a secant approximation to the matrix from iteration $i-1$ to $i$. That is, if we define a displacement increment

$$
\boldsymbol{\delta}^{(i)}={ }^{t+\Delta t} \boldsymbol{U}^{(i)}-{ }^{t+\Delta t} \boldsymbol{U}^{(i-1)}
$$

and an increment in the out-of-balance loads

$$
\boldsymbol{\gamma}^{(i)}=\left({ }^{t+\Delta t} \tilde{\boldsymbol{R}}^{(i-1)}-{ }^{t+\Delta t} \boldsymbol{F}^{(i-1)}\right)-\left({ }^{t+\Delta t} \tilde{\boldsymbol{R}}^{(i)}-{ }^{t+\Delta t} \boldsymbol{F}^{(i)}\right),
$$

then the updated matrix ${ }^{t+\Delta t} \hat{\boldsymbol{K}}^{(i)}$ should satisfy the quasi-Newton equation (see [13])

$$
{ }^{t+\Delta t} \hat{\boldsymbol{K}}^{(i)} \boldsymbol{\delta}^{(i)}=\gamma^{(i)}
$$


These quasi-Newton methods provide a compromise between the full re-formation of the stiffness matrix performed in the full Newton method and the use of a stiffness matrix from a previous configuration as is done in the modified Newton method. Among the quasi-Newton methods available the BFGS (Broyden-Fletcher-Goldfarb-Shanno) method appears to be most effective [13]-[15] and was recently proposed for finite element analysis by Matthies and Strang [15].

In the BFGS method the following procedure is employed in iteration $i$ to evaluate ${ }^{t+\Delta t} U^{(i)}$ and ${ }^{t+\Delta t} \hat{\boldsymbol{K}}^{(i)}$, where ${ }^{t+\Delta t} \hat{\boldsymbol{K}}^{(0)}={ }^{\tau} \hat{\boldsymbol{K}}$.

Step 1. Evaluate a displacement vector increment

$$
\Delta \bar{U}=\left({ }^{+\Delta t} \hat{\boldsymbol{K}}^{-1}\right)^{(i-1)}\left({ }^{t+\Delta t} \tilde{\boldsymbol{R}}^{(i-1)}-{ }^{t+\Delta t} \boldsymbol{F}^{(i-1)}\right) .
$$

This displacement vector defines a "direction" for the actual displacement increment.

Step 2. Perform a line search in the direction $\Delta \bar{U}$ to satisfy "equilibrium" in this direction. In this line search we evaluate the displacement vector

$$
{ }^{t+\Delta t} U^{(i)}={ }^{t+\Delta t} U^{(i-1)}+\beta \Delta \bar{U}
$$

where $\beta$ is a scalar multiplier, and we calculate the out-of-balance loads ${ }^{t+\Delta t} \tilde{\boldsymbol{R}}^{(i)}-{ }^{t+\Delta t} \boldsymbol{F}^{(i)}$ corresponding to these displacements. The parameter $\beta$ is varied until the component of the out-of-balance loads in the direction $\Delta \bar{U}$ as defined by the inner product $\Delta \bar{U}^{t}\left({ }^{t+\Delta t} \tilde{R}^{(i)}-{ }^{t+\Delta t} F^{(i)}\right)$ is approximately zero. This condition is satisfied when the following equation is satisfied for a convergence tolerance STOL:

$$
\Delta \overline{\boldsymbol{U}}^{t}\left({ }^{t+\Delta t} \tilde{\boldsymbol{R}}^{(i)}-{ }^{t+\Delta t} \boldsymbol{F}^{(i)}\right) \leq \mathrm{STOL} \Delta \overline{\boldsymbol{U}}^{t}\left({ }^{t+\Delta t} \tilde{\boldsymbol{R}}^{(i-1)}-{ }^{t+\Delta t} \boldsymbol{F}^{(i-1)}\right)
$$

The final value of $\beta$ for which eq. (30) is satisfied determines ${ }^{t+\Delta t} U^{(i)}$ using eq. (29). We can now calculate $\delta^{(i)}$ and $\gamma^{(i)}$ from eqs. (25) and (26) and proceed with the evaluation of the matrix update that satisfies eq. (27).

Step 3. Evaluate the correction to the coefficient matrix. In the BFGS method the updated matrix can be expressed in product form (see [25]):

$$
\left({ }^{t+\Delta t} \hat{K}^{-1}\right)^{(i)}=\boldsymbol{A}^{(i) t}\left({ }^{t+\Delta t} \hat{K}^{-1}\right)^{(i-1)} A^{(i)}
$$

where the matrix $A^{(i)}$ is an $n \times n$ matrix of the simple form

$$
\boldsymbol{A}^{(i)}=\boldsymbol{I}+\boldsymbol{v}^{(i)} \boldsymbol{w}^{(i) t}
$$

The vectors $v^{(i)}$ and $w^{(i)}$ are calculated from the known nodal point forces and displacements:

$$
\boldsymbol{v}^{(i)}=-\left[\frac{\delta^{(i) t} \gamma^{(i)}}{\delta^{(i) t+\Delta t} \hat{\boldsymbol{K}}^{(i-1)} \delta^{(i)}}\right]^{1 / 2}{ }^{t+\Delta t} \boldsymbol{K}^{(i-1)} \boldsymbol{\delta}^{(i)}-\boldsymbol{\gamma}^{(i)}
$$


and

$$
w^{(i)}=\frac{\delta^{(i)}}{\delta^{(i) t} \gamma^{(i)}}
$$

The vector ${ }^{t+\Delta t} \hat{\boldsymbol{K}}^{(i-1)} \boldsymbol{\delta}^{(i)}$ in eq. (33) is equal to $\beta\left[{ }^{t+\Delta t} \tilde{\boldsymbol{R}}^{(i-1)}-{ }^{t+\Delta t} \boldsymbol{F}^{(i-1)}\right]$ and was already computed.

It has been shown that the product defined in eq. (31) is positive definite and symmetric [15]. To avoid numerically dangerous updates, the condition number of the updating matrix $\boldsymbol{A}^{(i)}$ must be compared with some preset tolerance, and the updating is not performed if the condition number exceeds this tolerance. The eigenvalues of the matrix $I+v w^{t}$ are $\lambda=1$ (with multiplicity $n-1)$ and $\lambda=1+v^{t} w$. The condition number $c^{(i)}$ is therefore given by

$$
c^{(i)}=\left[\frac{\boldsymbol{\delta}^{(i) \mathrm{t}} \boldsymbol{\gamma}^{(i)}}{\boldsymbol{\delta}^{(i) t+\Delta t} \hat{\boldsymbol{K}}^{(i-1)} \boldsymbol{\delta}^{(i)}}\right]^{1 / 2} .
$$

A large condition number implies that the updated inverse matrix will be nearly singular. In our investigations we did not perform the matrix update when $c^{(i)}$ was larger than $10^{5}$.

Considering the actual computations involved, it should be recognized that if the matrix updates defined above are used, eq. (28) can be rewritten as

$$
\begin{aligned}
\Delta \vec{U}=(\boldsymbol{I} & \left.+\boldsymbol{w}^{(i-1)} \boldsymbol{v}^{(i-1) t}\right) \cdots\left(\boldsymbol{I}+\boldsymbol{w}^{(1)} \boldsymbol{v}^{(1) t}\right)^{\tau} \hat{\boldsymbol{K}}^{-1}\left(\boldsymbol{I}+\boldsymbol{v}^{(1)} \boldsymbol{w}^{(1) t}\right) \cdots\left(\boldsymbol{I}+\boldsymbol{v}^{(i-1)} \boldsymbol{w}^{(i-1) t}\right) \\
& \times\left[{ }^{t+\Delta t} \tilde{\boldsymbol{R}}^{(i-1)}{ }^{t+\Delta t} \boldsymbol{F}^{(i-1)}\right] .
\end{aligned}
$$

The form of eq. (36) enables the search direction to be computed without explicitly calculating the updated matrices or performing any additional costly matrix factorizations (as required in the full Newton method).

It is instructive to examine the convergence of the BFGS method in the analysis of a one-degree-of-freedom system. In this case the updating by the matrices $\boldsymbol{A}^{(i)}$ simply gives

$$
{ }^{t+\Delta t} \boldsymbol{K}^{(i)}=\frac{\gamma^{(i)}}{\delta^{(i)}}
$$

Fig. 4 illustrates how the BFGS method uses the secant approximation to converge to the equilibrium solution.

An important point when considering the BFGS method is its relatively good convergence behavior in the analysis of many problems encountered in finite element analysis. Theoretically, convergence is guaranteed [14], but a very large number of iterations may be required. In practice, the method may not converge because the line search is performed with too large a tolerance STOL (see eq. (30)) or the matrix updating is not carried out because of illconditioning. A relatively large tolerance for STOL is attractive in practice because the number of line searches, and thus solution cost, is reduced, and experience shows that, nevertheless, for many problems an accurate solution is obtained. Based on our present experience, we generally use $\mathrm{STOL}=0.5$ (see section 5). 


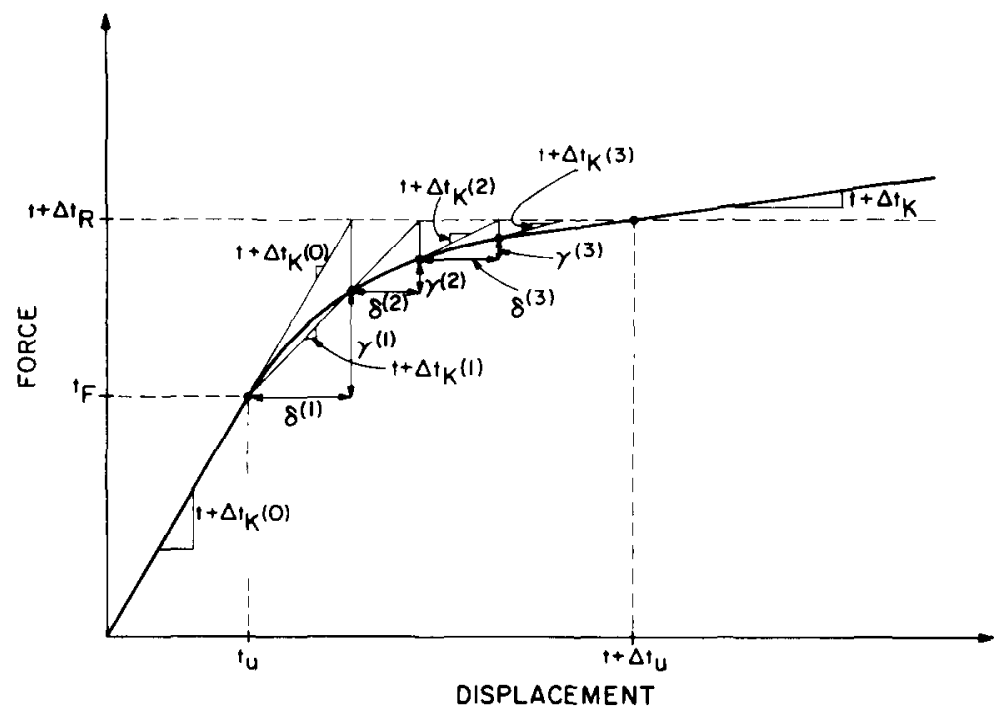

Fig. 4. Performance of the BFGS method in a one-dimensional example.

\section{Convergence criteria}

If an incremental solution strategy based on iterative methods is to be effective, realistic criteria should be used for the termination of the iteration. At the end of each iteration the solution obtained should be checked to see whether it has converged within preset tolerances or whether the iteration is diverging. If the convergence tolerance is too loose, inaccurate results are obtained, and if the tolerance is too tight, much computational effort is spent to obtain needless accuracy. Similarly, an ineffective divergence check can terminate the iteration when the solution is not actually diverging or force the iteration to search for an unattainable solution. Three solution variables available for termination criteria are: the displacements, the out-of-balance forces and the incremental internal energy.

Since we are seeking the displacement configuration corresponding to time $t+\Delta t$, it is natural to require that the displacements at the end of each iteration be within a certain tolerance of the true displacement solution. Hence, a realistic displacement convergence criterion is

$$
\left\|\frac{\Delta U^{(i)} \|_{2}}{\| t+\Delta t}\right\|_{2} \leq \epsilon_{D}
$$

where $\|a\|_{2}$ denotes the Euclidean norm of $a[16]$, and $\epsilon_{D}$ is some preset displacement tolerance. Unfortunately, $\left\|^{t+\Delta t} U\right\|_{2}$ is not known a priori and must be approximated.

One way to approximate $\left\|^{t+\Delta t} U\right\|_{2}$ is to use a convergence factor (see [12])

$$
q=\frac{\left\|\Delta \boldsymbol{U}^{(i)}\right\|_{2}}{\left\|\Delta \boldsymbol{U}^{(i-1)}\right\|_{2}}
$$


If the iteration is converging $(q<1)$ and the convergence factor for future iterations does not exceed the current convergence factor, then

$$
\left\|{ }^{t+\Delta t} \boldsymbol{U}-{ }^{t+\Delta t} \boldsymbol{U}^{(i-1)}\right\|_{2} \leq \sum_{k=1}^{\infty} q^{k}\left\|\Delta \boldsymbol{U}^{(i)}\right\|_{2}
$$

where ${ }^{t+\Delta t} U$ is the equilibrium displacement vector.

Since $q$ is less than one, we have

$$
\left\|{ }^{t+\Delta t} \boldsymbol{U}-{ }^{t+\Delta t} \boldsymbol{U}^{(i-1)}\right\|_{2} \leq \frac{q}{1-q}\left\|\Delta \boldsymbol{U}^{(i)}\right\|_{2}
$$

If the convergence factor is used to project ahead to the correct solution, then a displacement convergence criterion appears as

$$
\frac{q}{1-q}\left\|\Delta U^{(i)}\right\|_{2} \leq \epsilon_{D}\left\|^{r} U\right\|_{2}
$$

where $\|\boldsymbol{t} U\|_{2}$ is some previously calculated displacement norm, usually $\|\boldsymbol{U}\|_{2}$. Although this convergence criterion is effective in the analysis of a one-degree-of-freedom system, experience has shown that in general nonlinear analysis the convergence factor can be ill-behaved and is therefore not a reliable indicator of how the iteration is proceeding. In the analysis of a cantilever beam undergoing large deflections (see section 5.1) $q$ was found to be $0.016,9.5$, 0.08 and 0.66 in iterations $5,6,7$ and 8 .

A more reliable convergence criterion is based on the out-of-balance forces. A force convergence criterion requires that the norm of the out-of-balance load vector be within a preset tolerance $\epsilon_{F}$ of the original load increment:

$$
\left\|{ }^{t+\Delta t} \tilde{\boldsymbol{R}}^{(i)}-{ }^{t+\Delta t} \boldsymbol{F}^{(i)}\right\|_{2} \leq \boldsymbol{\epsilon}_{\boldsymbol{F}}\left\|^{t+\Delta t} \boldsymbol{R}-{ }^{t} \boldsymbol{F}-\boldsymbol{M} \cdot \ddot{\boldsymbol{U}}\right\|_{2}
$$

In order that the force convergence criterion does not become too restrictive for small load increments, the maximum initial load increment $\left\|^{t+\Delta t} \boldsymbol{R}-{ }^{t} \boldsymbol{F}-\boldsymbol{M}^{t} \ddot{\boldsymbol{U}}\right\|_{2}^{(\max )}$ is preferably used in eq. (43) instead of $\left\|^{t+\Delta t} \boldsymbol{R}-{ }^{t} \boldsymbol{F}-\boldsymbol{M}^{t} \ddot{\boldsymbol{U}}\right\|_{2}$, where the superscript (max) denotes the maximum value ever calculated during the solution.

The major disadvantages in using a force check are that inconsistencies in units can appear in the force vector (e.g. forces and moments in beam elements [24]) and that the displacement solution does not enter the termination criterion. As an illustration of the latter difficulty, consider an elastic-plastic truss with a very small strain-hardening modulus entering the plastic region. In this case the out-of-balance loads may be very small while the displacements may still be grossly in error.

In order to provide some indication of when both the displacements and forces are near their equilibrium values, the increment in internal energy during each iteration (i.e. the amount of work done by the out-of-balance loads on the displacement increments) can be compared to the initial internal energy increment. Convergence is assumed to be reached when 


$$
\Delta U^{(i) t}\left({ }^{t+\Delta t} R-{ }^{t+\Delta t} F^{(i-1)}-M^{t+\Delta t} \ddot{U}^{(i-1)}\right) \leq \epsilon_{E} \Delta U^{(1) t}\left({ }^{t+\Delta t} R-{ }^{t} \boldsymbol{F}-M^{t} \ddot{U}\right),
$$

where $\epsilon_{E}$ is the preset energy tolerance.

The various characteristics of termination criteria make it difficult to recommend any one check for all nonlinear analyses. However, it appears that a combination of force and energy checks (eqs. (43) and (44)) provide the most effective convergence criteria because increments in both terms tend to zero near the solution, and together they provide some measure of the accuracy of both displacements and forces (see section 5.1 for comparison with a displacement check). Also, the energy check (eq. (44)) with $\epsilon_{E}=1$ is recommended as a check for divergence. Finally, the preset tolerances should reflect the characteristics of the problem. "Stiffening" structures require a tight force tolerance, while "softening" structures require a tight displacement or energy tolerance. To see the effects of tolerances on the accuracy of an analysis, see section 5.6.

\section{Sample solutions}

The determination of the most effective approach to a general nonlinear analysis is at present largely a matter of experience on the part of the analyst. In this section some demonstrative solutions are presented that illustrate the points made previously and give some insight into how the solution of a specific kind of nonlinear problem should be approached.

All sample problems were solved using the computer program ADINA [26], in which the nonlinear solution algorithms discussed above are available. Unless otherwise noted, the convergence criteria for equilibrium iterations were the force and energy checks in eqs. (43) and (44) using tolerances of:

$$
\epsilon_{F}=0.1, \quad \epsilon_{D}=0.001, \quad \epsilon_{E}=10 \epsilon_{F} \epsilon_{D}
$$

The line search tolerance STOL for the BFGS method was set to 0.5 for all analyses.

\subsection{Static large displacement analysis of a cantilever}

The cantilever shown in fig. 5 was subjected to a uniformly distributed load. The finite element mesh consists of five 8-node plane stress elements of isotropic linear elastic material. The problem is geometrically nonlinear and the cantilever stiffens with increasing displacements. The total Lagrangian formulation was used.

A static analysis of the cantilever was carried out using different load increments. The relationship between the load increment sizes and the number of iterations plus the computer times used are shown in table 1 . In each case the load increment was constant throughout the analysis. The BFGS method converges for all load increments and, as shown in fig. 5, is always in close agreement with the analytical solution by Holden [27]. Convergence was sometimes slow, for example requiring 78 iterations in one step when $\Delta K=5.0$, but it is important to note that the BFGS method does allow large load increments to be taken with no divergence in the iteration.

To demonstrate the performance of the modified Newton method with Aitken acceleration 




Fig. 5. Large displacement analysis of a cantilever.

Table 1. Comparison of iterative schemes and load increments in the static large displacement analysis of a cantilever

\begin{tabular}{|c|c|c|c|c|c|}
\hline \multirow[b]{2}{*}{$\Delta K$ per step } & \multicolumn{4}{|c|}{-BFGS method. } & \multirow{2}{*}{$\begin{array}{l}\text { Modified } \\
\text { Newton } \\
0.5\end{array}$} \\
\hline & 0.5 & 1.0 & 2.0 & 5.0 & \\
\hline No. of load steps & 20 & 10 & 5 & 2 & 37 \\
\hline Total no. of iterations & 146 & 104 & 78 & 104 & 883 \\
\hline$w_{\text {tip }} / L$ at maximum load & 0.685425 & 0.685429 & 0.685437 & 0.685285 & 0.685389 \\
\hline $\begin{array}{l}\text { Computer time* required for } \\
\text { equilibrium iteration }\end{array}$ & 4.00 & 3.34 & 3.08 & 4.98 & 15.74 \\
\hline $\begin{array}{l}\text { Total computer time* for } \\
\text { solution }\end{array}$ & 6.05 & 4.35 & 3.57 & 5.17 & 17.83 \\
\hline
\end{tabular}

${ }^{*}$ Computer times are in seconds on a Control Data Cyber 175.

and the divergence procedure on a slowly stiffening problem, the cantilever problem was analyzed with a constant increment of 0.5 in the load parameter $K$. In each step from $K=0.0$ to $K=3.0$ it was necessary to use the divergence procedure to subdivide the load increment in order to obtain convergence with the modified Newton method. Slow convergence (10 to 15 
iterations) was then encountered up to $K=7.5$, where again the divergence procedure was needed. From $K=8.0$ to the final load level of $K=10.0$ rapid convergence ( 2 to 5 iterations) was observed. The number of iterations and the total solution time for the modified Newton method were significantly higher than they were in any of the BFGS runs. The results indicate however that the modified Newton method can be made to converge in normally divergent geometric stiffening problems when Aitken acceleration and the divergence procedure are employed.

Finally, this problem was used to compare the displacement-based convergence tolerance with the energy-based tolerance. As shown in table 2, the energy tolerance is both more effective and less erratic in the number of iterations required, probably because of the erratic behavior of the convergence factor, as mentioned in section 4 . There is no difference in the calculated tip deflections at any load level.

\subsection{Elastic-plastic static analysis of a thick-walled cylinder}

The thick-walled cylinder shown in fig. 6 was subjected to one cycle of pressurization and depressurization. Plane strain conditions were assumed, and the cylinder was modeled with four 8-node axisymmetric elements.

The material was assumed to be elastic-perfectly plastic and to obey the von Mises yield condition. Since displacements and strains are small, the analysis was carried out for material nonlinearities only. The problem includes both softening behavior (as plasticity develops in the cylinder) and sudden stiffening (as the cylinder is unloaded). Since the problem involves radial loading, the size of the load increment does not affect the plasticity solution.

Table 2. Comparison of displacement convergence criterion with energy convergence criterion in the large deflection static analysis of a cantilever

\begin{tabular}{ccccc}
\hline $\begin{array}{c}\epsilon_{F}=0.1 \\
\text { Load } \\
\text { parameter }\end{array}$ & \multicolumn{2}{c}{$\epsilon_{\nu}=0.001$} & \multicolumn{2}{c}{$\begin{array}{c}\epsilon_{E}=0.001 \\
w_{\text {tip }} / L\end{array}$} \\
$K$ & $\begin{array}{c}\text { Do. of iterations } \\
\text { Displacement } \\
\text { criterion }\end{array}$ & $\begin{array}{c}\text { Energy } \\
\text { criterion }\end{array}$ & $\begin{array}{c}\text { Displacement } \\
\text { criterion }\end{array}$ & $\begin{array}{c}\text { Energy } \\
\text { criterion }\end{array}$ \\
\hline 0.5 & 10 & 10 & 0.063 & 0.063 \\
1.0 & 15 & 9 & 0.124 & 0.124 \\
1.5 & 11 & 9 & 0.183 & 0.183 \\
2.0 & 11 & 9 & 0.238 & 0.238 \\
2.5 & 10 & 9 & 0.290 & 0.290 \\
3.0 & 9 & 8 & 0.338 & 0.338 \\
3.5 & 9 & 8 & 0.382 & 0.382 \\
4.0 & 11 & 8 & 0.422 & 0.422 \\
4.5 & 7 & 8 & 0.458 & 0.458 \\
5.0 & 7 & 7 & 0.491 & 0.491 \\
& & & & \\
Total number & 100 & 85 & & \\
of iterations & & & & \\
\hline
\end{tabular}

Displacement criterion consists of eq. (41)

Energy criterion consists of eq. (44)

In both cases eq. (43) also had to be satisfied 

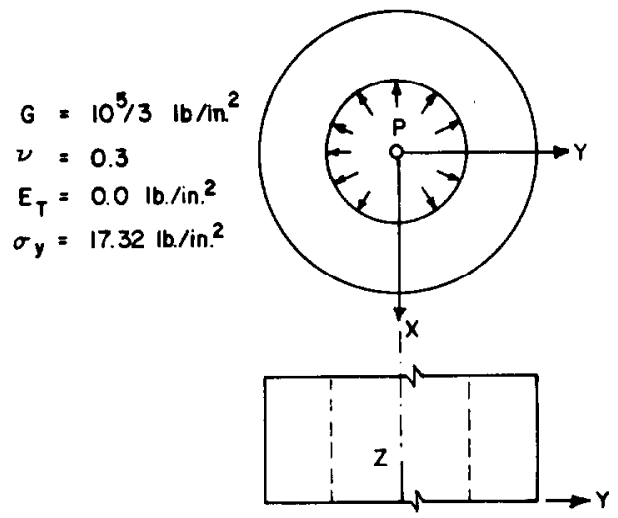

TOP AND SIDE VIEWS


ENLARCED TOP VIEW AND AXISYMMETRIC MESH

ELASTIC -PERFECTLY PLASTIC MATERIAL

PLANE STRAIN CONDITIONS

VON-MISES YIELD CONDITION

Fig. 6. Finite element analysis of an elastic-plastic thick-walled cylinder.

To test the performance of various iterative methods when large load increments are taken, the cylinder was pressurized to its peak pressure $(99 \%$ of the collapse load) in one step and then depressurized in the next step. Since the problem involves softening, the displacement tolerance $\epsilon_{D}$ was set to 0.00001 , one-hundreth of the normally used value.

When the modified Newton method without Aitken acceleration was used, 56 iterations were required for convergence upon loading, and the solution diverged on unloading. Using the Aitken acceleration, only 27 iterations were required for convergence upon loading. To converge in the unloading, the divergence procedure was employed-this converged in two iterations. The use of Aitken acceleration did not contribute significantly to the solution time per iteration; the time required for the run with acceleration was about half the time required for the run without acceleration.

The BFGS method required only six iterations for convergence in the loading part of the analysis, but the cost was equivalent to the Aitken analysis, primarily due to tape processing costs. In all analyses the displacements were within $2 \%$ of the solution obtained by Hodge and White [28].

\subsection{Static analysis of a reinforced concrete beam}

The simply supported reinforced concrete beam in fig. 7 was subjected to two concentrated loads. The analysis used ten 6-node plane stress, concrete elements and five steel truss elements. Only material nonlinear response was assumed; i.e. large displacement effects were neglected.

The load-deflection curves calculated using both the BFGS method and no equilibrium iterations are shown in fig. 8, where also the results of Suidan and Schnobrich [29] are given. The load histories used for each ADINA analysis are also shown in the figure. Using the BFGS method, slow convergence was observed when the concrete entered the nonlinear 

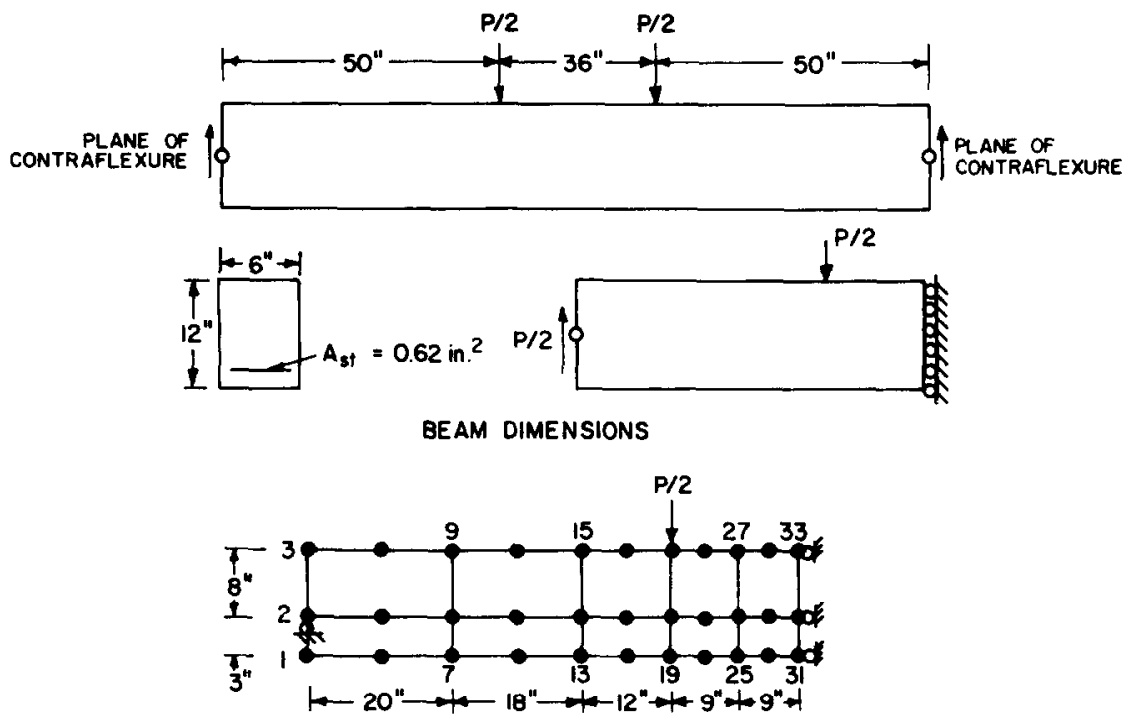

FINITE ELEMENT IDEALIZATION

MATERIAL PROPERTIES:

$$
\begin{aligned}
& \sigma_{c}=-3740 \mathrm{psi} \\
& \sigma_{f}=458 \mathrm{psi} \\
& \sigma_{y \text { steel }}=44000 \mathrm{psi} \\
& E_{\sigma_{\text {concrote }}}=6100 \mathrm{ksi} \\
& \nu=0.2 \\
& E_{\text {steel }}=30000 \mathrm{ksi} \\
& E_{f_{\text {steel }}}=300 \mathrm{ksi}
\end{aligned}
$$

$$
\begin{aligned}
& \sigma_{u}=-3225 \mathrm{psi} \\
& \theta_{0}=-.003 \mathrm{in} / \mathrm{in} . \\
& \theta_{c}=-.002 \mathrm{in} / \mathrm{in} . \\
& \rho_{\text {concrete }}=0.2172 \times 10^{-3} \text { slugs } / \mathrm{in}^{3} . \\
& \rho_{\text {steel }}=0.7339 \times 10^{-3} \text { shuss } / \mathrm{in}^{3} .
\end{aligned}
$$

Fig. 7. Finite element analysis of a reinforced concrete beam.

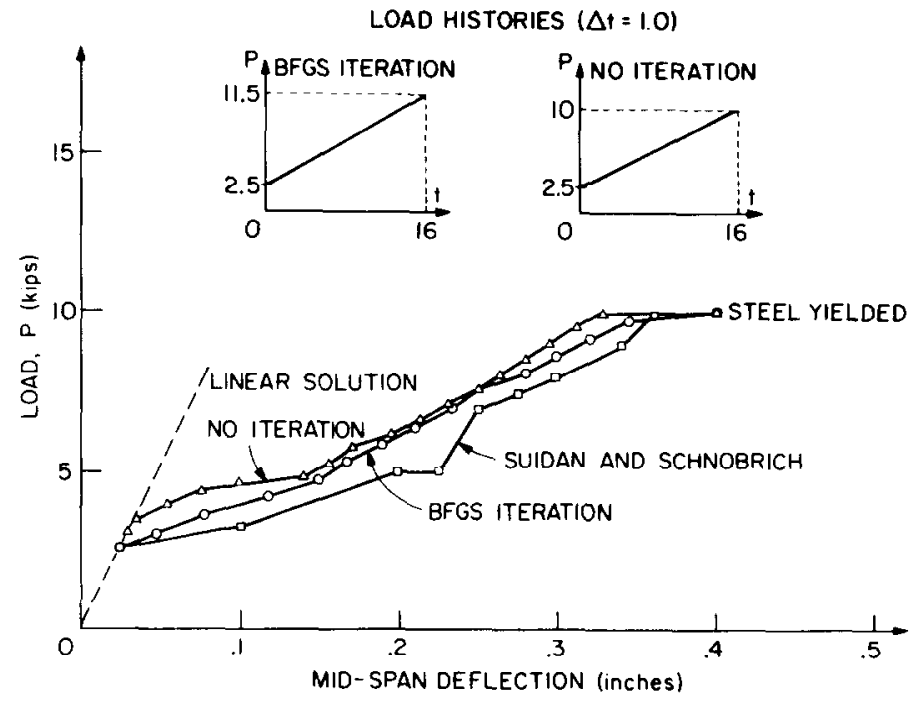

Fig. 8. Load-deflection response for the concrete beam. 
region and when the steel yielded. The number of iterations required for convergence in each load step is shown in table 3 . When the load increment size was doubled, convergence was not obtained in the first step after 100 BFGS iterations. When the modified Newton method was used, the iteration would not converge when 64 equal load steps were used to describe the entire load history. The severe nature of the nonlinearity as the concrete begins to crack is probably responsible for the difficulty in establishing equilibrium when the nonlinear region is first entered.

The solution obtained using no iteration shows the largest deviation from the equilibrium curve when nonlinearities are first encountered and when the collapse load is approached. Further refinement of the load increment would result in better agreement between the solution using no iteration and the equilibrium solution. When complex nonlinear material models such as the concrete model are employed and the cost for each iteration becomes high, it can be more cost-effective to abandon iteration and use a smaller load increment.

\subsection{Large deflection analysis of a cable structure}

The cable shown in fig. 9, modeled using 10 linear elastic truss elements, was subjected to a uniformly distributed load simulating the cable weight. The upper end was then displaced from its original location to a point directly above the cable anchor point. The updated Lagrangian formulation was used in the analysis. Two hundred steps were employed to model the load history.

It is important to maintain equilibrium in all steps in this analysis because the nonlinear incremental analysis requires that all truss elements remain in tension to keep the stiffness

Table 3. Number of iterations required for convergence of the BFGS method in the static analysis of a reinforced concrete beam

\begin{tabular}{clc}
\hline $\begin{array}{c}\text { Load } \\
\text { step }\end{array}$ & $\begin{array}{c}\text { Load level } \\
\boldsymbol{P}\end{array}$ & $\begin{array}{c}\text { No. of } \\
\text { iterations }\end{array}$ \\
\hline 1 & 3.0625 & 11 \\
2 & 3.625 & 15 \\
3 & 4.1875 & 19 \\
4 & 4.75 & 3 \\
5 & 5.3125 & 3 \\
6 & 5.875 & 10 \\
7 & 6.4375 & 3 \\
8 & 7.0 & 3 \\
9 & 7.5625 & 6 \\
10 & 8.125 & 13 \\
11 & 8.6875 & 3 \\
12 & 9.25 & 3 \\
13 & 9.8125 & 6 \\
14 & 10.375 & 15 \\
15 & 10.9375 & 34 \\
16 & 11.5 & 6 \\
\hline
\end{tabular}




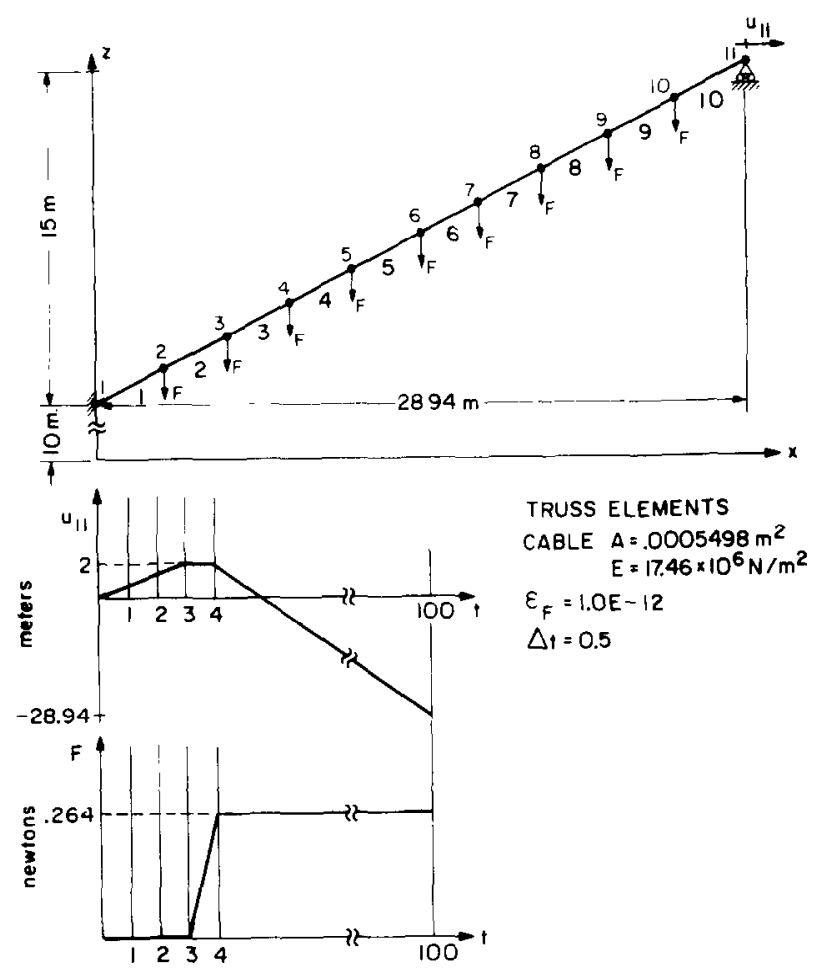

Fig. 9. Finite element analysis of a cable structure.

matrix positive definite. Also, the initially large pretension in the cable during the first few load steps makes the right-hand side of the force check (eq. (43)) large. As the forces in the trusses become small for large cable deflections, the force tolerance needs to be very tight to keep the force check accurate. To complete the analysis with the given load history it was necessary to set $\epsilon_{F}$ to $1.0 \times 10^{-12}$ and use the BFGS method. Only 2 to 3 iterations were required to establish equilibrium for most load steps; however, 10 to 15 iterations were required for certain load steps scattered throughout the analysis.

Fig. 10 shows the cable configuration for two locations of the cable end point. The analysis results compare favorably with experimental results [30] even though a coarse finite element mesh is used to model the cable curvature.

\subsection{Dynamic analysis of a pipe whip problem}

The pipe whip problem illustrated in fig. 11 was modeled using 6 beam elements of a pipe cross-section that impinges on an elastic-plastic stop. The trapezoidal rule for time integration was employed in this analysis. When the gap closes, there is a large stiffening effect that renders the convergence of the modified Newton iteration difficult.

Fig. 12 shows the predicted displacement response when the BFGS method was used with time steps $\Delta t$ of $0.0001,0.0002,0.001$ and $0.002 \mathrm{sec}$ and $\epsilon_{F}=0.0001$. As expected, it can be seen that increasing the time step results in a loss of accuracy, even though equilibrium has been established at all time steps. This loss of accuracy is due to the errors associated with the 


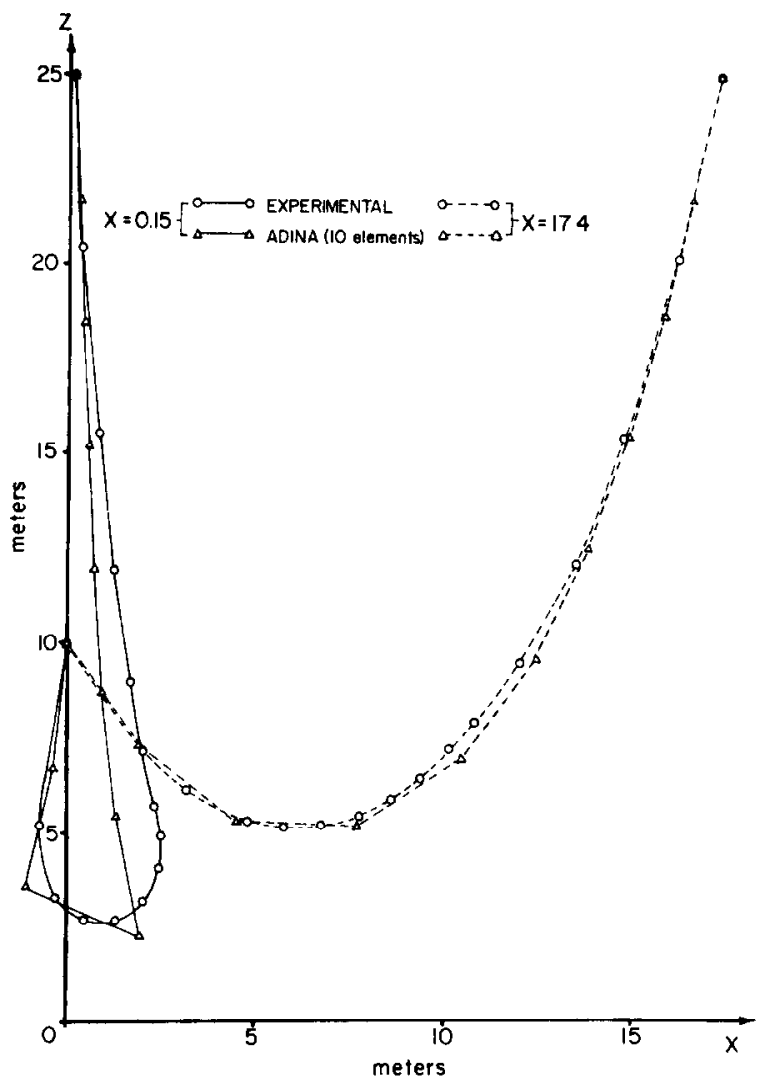

Fig. 10. Configurations of the cable for large displacements.

time integration scheme [16], [17]. The use of iteration however does assure the accurate solution of the timewise discretized equilibrium equations. In the analysis the average number of iterations per step was $1,2,3$ and 4 , using the time steps given above.

\subsection{Dynamic analysis of a simple pendulum}

A simple pendulum idealized as a truss element with a concentrated mass at its end was analyzed using the trapezoidal rule and the BFGS iteration. The pendulum was released from a horizontal position, and the response was calculated for about one period of oscillation.

Fig. 13 shows the response predicted with the time step $\Delta t=0.1 \mathrm{sec}$, using two different energy convergence tolerances $\epsilon_{E}$. It is seen that using $\epsilon_{E}=10^{-3}$, the solution is very inaccurate, and there is an energy loss in the analysis as the pendulum only returns to an angle of about $57^{\circ}$. With a tighter tolerance $\left(\epsilon_{E}=10^{-7}\right)$ the response is predicted correctly. Averages of four and six equilibrium iterations per time step were required with $\epsilon_{F}=10^{-1}, \epsilon_{E}=10^{-3}$ and $\epsilon_{F}=10^{-4}, \epsilon_{E}=10^{-7}$, respectively.

This analysis illustrates the importance of equilibrium iteration in dynamic analyses and the importance of using a sufficiently tight convergence tolerance. 


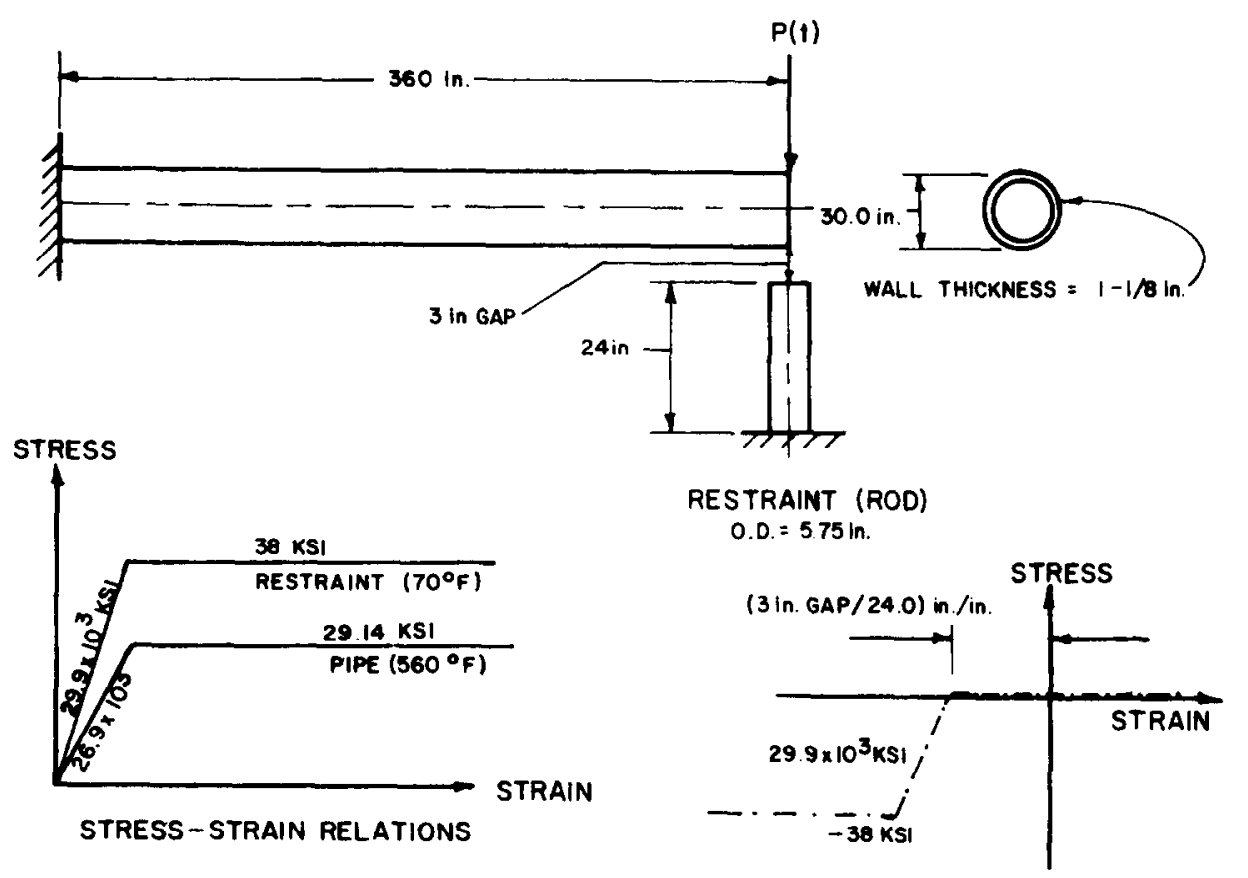

STRESS-STRAN CURVE FOR TRUSS ELEMENT

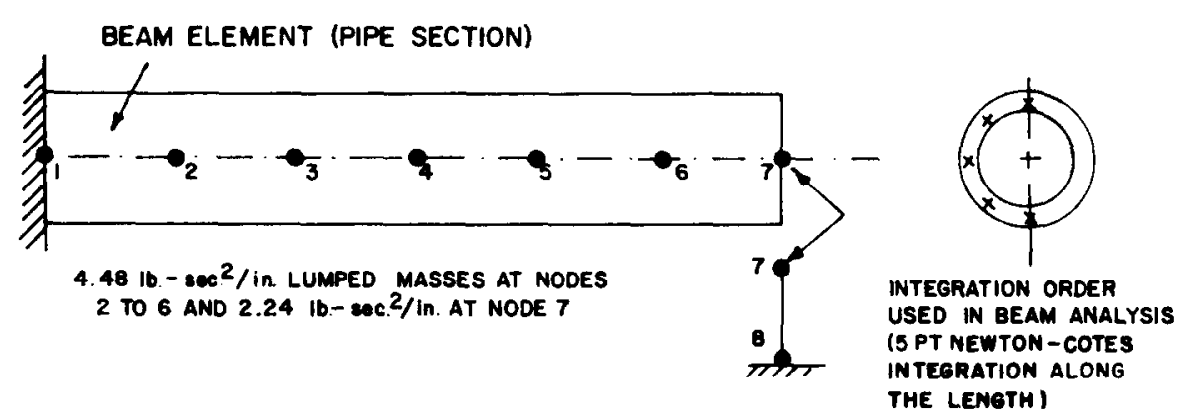

Fig. 11. Finite element analysis of a pipe whip problem.

\section{Conclusions}

Considering the solution of nonlinear finite element equations, the ultimate aim in many respects is the development of a solution scheme that automatically adjusts load increments, time-step sizes, convergence tolerances and other parameters to produce an accurate and cost-effective solution. However, until a completely self-adaptive scheme is available, some rational guidelines must be provided to assist the analyst in solving nonlinear finite element equations effectively with the methods that are already available.

\subsection{Static analysis}

In the initial analysis of a static problem, where the character of the response is unknown, it is recommended that the analysis first be performed without equilibrium iterations, but in 


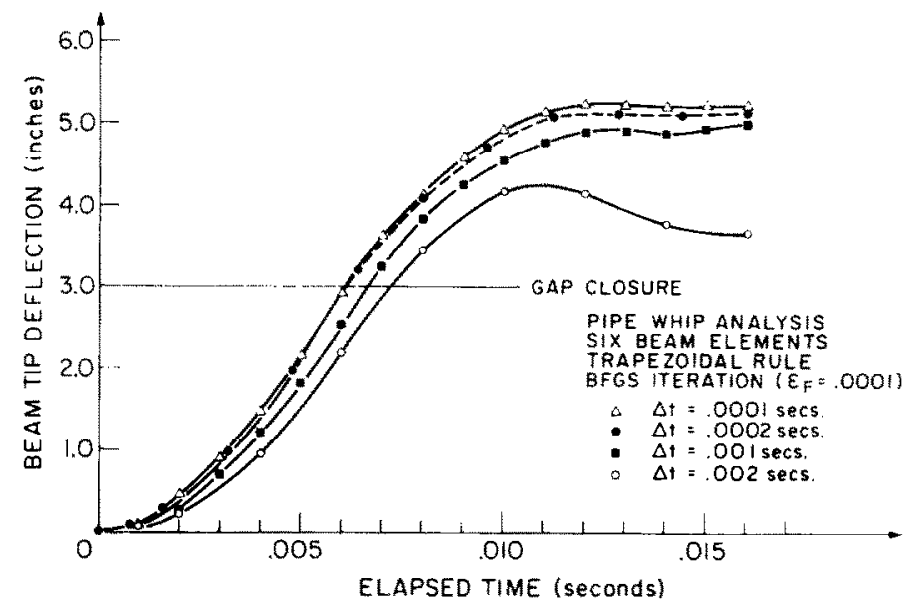

Fig. 12. Predicted displacement response in the pipe whip analysis for four different time step sizes.



Fig. 13. Analysis of a simple pendulum with the trapezoidal rule.

general the stiffness matrix should be re-formed at the beginning of each load step. Some reasonable estimate for the load increments should be used based on a knowledge of the nonlinear behavior or by analogy with a similar analysis. After an estimate of the solution is obtained, the accuracy of the analysis must be verified. There are two possible approaches for this verification. One approach is to significantly decrement the load steps where nonlinear response was encountered and compare the two solutions. The accuracy of the first analysis is confirmed if in the second solution the same response has been calculated. Further decrementing of the load steps is required if a significant difference in the solutions is observed. The second method of verifying the accuracy of the original analysis is to compare the original response with the response predicted using equilibrium iterations in the nonlinear portions of the solution. 
The selection of either the modified Newton method or the BFGS method for equilibrium iteration should depend on the type of nonlinearity encountered in the solution. If the stiffness of the structure is decreasing, particularly in analyses with material softening, the modified Newton method appears to be the more cost-effective. Aitken acceleration and a divergence procedure should in most cases be used with the modified Newton method since these do not contribute significantly to the solution cost and generally improve the performance of the modified Newton method. However, when complex material nonlinearities are present (e.g. the concrete beam in section 5.3), the BFGS method is preferable. In problems involving slow (geometric) stiffening, the load increment size drastically alters the iteration performance. In these analyses the use of the BFGS method is recommended with reasonably small load steps because the divergence procedure used with the modified Newton method has been found to be less effective in the analysis of slow stiffening response.

In using either equilibrium iteration method it should be recognized that the cost of an analysis can be high when many iterations are required because the evaluation of the force vector ${ }^{t+\Delta t} \boldsymbol{F}^{(i)}$ can be costly, particularly so in complex material nonlinear analysis. In general, a load step size should be small enough to allow convergence within a maximum of about 20 iterations. Also, the path-dependence of some material models should be considered in choosing the proper load increments. Finally, in any analysis involving iteration the convergence criteria must be carefully chosen to reflect the characteristics of the problem.

\subsection{Dynamic analysis}

Considering dynamic analysis, it is recommended that equilibrium iteration always be used, but it may not be necessary to calculate a new stiffness matrix in each time step. The inertia forces present in dynamic analysis tend to smooth out any sudden nonlinearities-this tends to give rapid convergence in the iteration. Because of the frequent occurrence of slow stiffening and the small number of iterations generally required in dynamic analysis it is recommended that the BFGS method be employed.

The recommendations summarized above for the solution of nonlinear finite element equations represent our experience with currently existing techniques. A more effective solution procedure may well be available for the analysis of specific nonlinear problems [2], [19], [31]; however, our recommendations pertain to the solution of the general nonlinear finite element equations considered in the paper. These recommendations must necessarily be revised as new research results become available, and such results must surely be expected because the development of more efficient methods for nonlinear analysis is at present a very important and much-researched issue.

\section{Acknowledgment}

We would like to thank G. Strang and $\mathrm{H}$. Matthies for valuable discussions on the solution of nonlinear finite element equations. We are grateful to the ADINA users group for the financial support of our work in computational mechanics. 


\section{References}

[1] W. Pilkey, K. Saczalski and H. Schaeffer (eds.), Structural Mechanics Computer Programs: surveys, assessments, and availability (Univ. Press of Virginia, Charlottesville, 1974).

[2] C.A. Felippa, Procedures for computer analysis of large nonlinear structural systems, Proceedings, International Symposium on Large Engineering Systems, Univ. Manitoba, Winnipeg, Canada, Aug. 1976.

[3] J.H. Argyris, J.St. Doltsinis and K.J. Willam, New developments in the inelastic analysis of quasistatic and dynamic problems, in: Méthodes numériques dans les sciences de l'ingénieur, Dunod technique bordas, Paris, 1979, 77-93.

[4] K.J. Bathe, E. Ramm and E.L. Wilson, Finite element formulations for large deformation dynamic analysis, Int. J. Numer. Meth. Eng. 9 (1973) 353-386.

[5] K.J. Bathe, Static and dynamic geometric and material nonlinear analysis using ADINA, Rep. 82448-2 (Acoustics and Vibration Lab., Mech. Eng. Dept., MIT, May 1976 (rev. May 1977)).

[6] K.J. Bathe, S. Bolourchi, S. Ramaswamy and M.D. Snyder, Some computational capabilities for nonlinear finite element analysis, J. Nuclear Eng. and Design 46 (1978) 429-455.

[7] K.J. Bathe, An assessment of current solution capabilities for nonlinear problems in solid mechanics, in: Numerical methods for partial differential equations III (Academic Press, 1976) 117-164.

[8] K.J. Bathe, Finite element formulation, modeling and solution of nonlinear dynamic problems, in: Numerical methods for partial differential equations (Academic Press, 1979) 1-40.

[9] T. Belytschko and D.F. Schoeberle, On the unconditional stability of an implicit algorithm for nonlinear structural dynamics, J. Appl. Mech. 42 (1975) 865-869.

[10] T.J.R. Hughes, T. Caughey and W. Liu, Finite-element methods for nonlinear elastodynamics which conserve energy, J. Appl. Mech. 45 (1978) 366-370.

[11] K.J. Bathe and A. Cimento, On the time integration of nonlinear dynamic response, in: Méthodes numériques dans les sciences de l'ingénieur, Dunod technique bordas, Paris, 1979, 95-105.

[12] G. Dahlquist and A. Bjorck, Numerical methods (Prentice-Hall, 1974).

[13] W. Murray (ed.), Numerical methods for unconstrained optimization (Academic Press, 1972).

[14] J.E. Dennis, Jr., A brief survey of convergence results for quasi-Newton methods, SIAM-AMS Proceedings 9 (1976) 185-200.

[15] H. Matthies and G. Strang, The solution of nonlinear finite element equations, Int. J. Numer. Meth. Eng., in press.

[16] K.J. Bathe and E.L. Wilson, Numerical methods in finite element analysis (Prentice-Hall, 1976).

[17] T.J.R. Hughes, Stability, growth and decay of energy of the average acceleration method in nonlinear structural dynamics, J. Computers and Structures 6 (1976) 313-324.

[18] K.J. Bathe and W. Hahn, On transient analysis of fluid-structure systems, J. Computers and Structures 10 (1978) 383-391.

[19] J.A. Stricklin, W.E. Haisler and W.A. von Riesemann, Evaluation of solution procedures for material and/or Geometrically nonlinear structural analysis, AIAA J. 11 (1973) 292-299.

[20] A.C. Aitken, The evaluation of the latent roots and latent vectors of a matrix, Proc. Roy. Soc. Edinburgh 57 (1937) 269-304.

[21] E. Boyle and A. Jennings, Accelerating the convergence of elastic-plastic stress analysis, Int. J. Numer. Meths. Eng. 7 (1973) 232-235.

[22] B. Irons and R. Tuck, A version of the Aitken accelerator for computer iteration, Int. J. Numer. Meths. Eng. 1 (1969) 275-277.

[23] W. Schmidt, Adaptive step size selection for use with the continuation method, Int. J. Numer. Meths. Eng. 12 (1978) 677-694.

[24] P.G. Bergan, G. Horrigmoe, B. Kräkeland and T.H. Soreide, Solution techniques for non-linear finite element problems, Int. J. Numer. Meths. Eng. 12 (1978) 1677-1696.

[25] K. Brodlie, A. Gourlay and J. Greenstadt, Rank-one and rank-two corrections to positive definite matrices expressed in product form, J. Inst. Math. Appl. 11 (1973) 73-82.

[26] K.J. Bathe, ADINA-a finite element program for automatic dynamic incremental nonlinear analysis, Rep. 82448-1 (Acoustics and Vibration Lab., Mech. Eng. Dept., MIT, May 1975 (rev. Dec. 1978)).

[27] J.T. Holden, On the finite deflections of thin beams, Int. J. Solids Struct. 8 (1972) 1051-1055. 
[28] P. Hodge and S. White, A quantitative comparison of flow and deformation theories of plasticity, J. Appl. Mech. 17 (1950) 180-184.

[29] M. Suidan and W. Schnobrich, Finite element analysis of reinforced concrete, J. Struct. Div. ASCE (1967) 235-254.

[30] Dumay, COFLEXIP, Paris, private communication.

[31] J.H. Argyris, L.E. Vaz and K.J. Willam, Improved solution methods for inelastic rate problems, J. Comp. Meths. Appl. Mech. Eng. 16 (1978) 231-277. 